\title{
Degenerate Sub-keV fermion dark matter from a solution to the Hubble tension
}

\author{
Gongjun Choi ${ }^{1,{ }^{* *}}$ Motoo Suzuki, ${ }^{1, \dagger}$ and Tsutomu T. Yanagida ${ }^{1,2, \$}$ \\ ${ }^{1}$ Tsung-Dao Lee Institute, Shanghai Jiao Tong University, Shanghai 200240, China \\ ${ }^{2}$ Kavli IPMU (WPI), UTIAS, The University of Tokyo, 5-1-5 Kashiwanoha, \\ Kashiwa, Chiba 277-8583, Japan
}

(Received 11 February 2020; accepted 1 April 2020; published 15 April 2020)

\begin{abstract}
We present a dark sector model addressing both the Hubble tension and the core-cusp problem. The model is based on a hidden Abelian gauge symmetry group with some chiral fermions required by the anomaly cancellation conditions, producing a candidate for the decaying fermion dark matter as a solution to the Hubble tension. Moreover, the sub-keV mass regime and the thermal history of the dark sector help the dark matter candidate resolve the core-cusp problem occurring in the standard $\Lambda$ cold dark matter cosmology.
\end{abstract}

DOI: 10.1103/PhysRevD.101.075031

\section{INTRODUCTION}

The presence of dark matter (DM) becomes an unquestionable fact thanks to various observational evidences. Nevertheless, its nature still remains unclear. So far, at least three physical features of DM are known, i.e., its stability, nonzero mass, and very weak interaction with the ordinary matter. Unfortunately, however, as for the three aspects of DM, none was examined clearly thus far. It still remains unanswered whether its stability is permanent or effective only for the time scale of the age of the Universe today. Also, the uncertainty in a possible DM mass has not been narrowed down yet, ranging from $\mathcal{O}\left(10^{-22}\right) \mathrm{eV}$ for the ultralight bosonic DM [1,2] to $\mathcal{O}(10) \mathrm{M}_{\odot}$ for primordial black holes ${ }^{1}$ or a macroscopic compact halo object. Above all, lacking knowledge about nongravitational interaction, which DM does, we are still even unaware of if DM is, either of directly or indirectly, coupled to or totally decoupled from the standard model (SM) sector.

In an effort to address the aforementioned questions, we give a special attention to two arguments based on cosmological and astrophysical phenomena. The recently raised Hubble tension is one of them. This regards the discrepancy reaching $\sim 4 \sigma$ level [4] between a local measurement of the Hubble expansion rate $\left(H_{0}\right)$ [5-8] and that inferred from the

\footnotetext{
*gongjun.choi@gmail.com

m0t@icrr.u-tokyo.ac.jp

*tsutomu.tyanagida@ipmu.jp

${ }^{1}$ See, e.g., [3] for the possibility of primordial black holes as all dark matter.

Published by the American Physical Society under the terms of the Creative Commons Attribution 4.0 International license. Further distribution of this work must maintain attribution to the author(s) and the published article's title, journal citation, and DOI. Funded by SCOAP ${ }^{3}$.
}

cosmic microwave background (CMB) observation [9]. While unknown systematics may affect the discrepancy [10-12], it could be a clue for a nature of DM. In particular, a decaying DM (DDM) solution $[13]^{2}$ to the tension amongst several resolutions proposes a possibility that the decay of DM with a lifetime $\sim 35$ Gyrs can relieve the tension. ${ }^{3}$ The advanced starting of the dark energy dominated era due to DM decay enables the faster expansion of the Universe at late times $[z \simeq \mathcal{O}(0.1)]$ and eventually the Hubble expansion rate at the recombination era determined by $\mathrm{CMB}$ data can evolve to a value close to the SHOES measurement of $H_{0} \simeq 73-74 \mathrm{Km} / \mathrm{sec} / \mathrm{Mpc}$ [6]. Inspired by this, we take a DM scenario with a finite lifetime greater than the current age of the Universe as far as the question about stability of $\mathrm{DM}$ is concerned.

On the other hand, whereas the correlation of structures on the large scale is in excellent accord with predictions from the Lambda cold dark matter $(\Lambda \mathrm{CDM})$ paradigm (cosmological constant + cold dark matter), discrepancies between results from simulations based on $\Lambda \mathrm{CDM}$ (e.g., cuspy halos [24], too many subhalos [25,26], and dense cores [27]) and the experimental observations on galactic scales may be hinting for a nature of DM distinct from CDM (for a review, see [28]). Particularly, from the disagreement between the cuspy DM density profile for galaxies predicted by $\Lambda \mathrm{CDM}$ simulations [29-31] and observations of rotation curves for low mass galaxies [32-35], there arises the so-called cusp-core problem. As one of the resolutions to the problem, the presence of degenerate fermion DM with sub-keV mass

\footnotetext{
${ }^{2}$ For an exemplary particle physics model for the DDM solution to the Hubble tension, see $[14,15]$.

${ }^{3}$ Other scenarios proposed to solve the tension include, for example, dark radiation $[5,16,17]$, dark energy at early time [18-21], interacting dark energy [22,23], etc.
} 
has been suggested in [36-41]. The quantum pressure applied by a degenerate fermion gas can counterbalance the gravity to prevent gravitational collapse, yielding cored DM profiles in dwarf spheroidal (dSphs) galaxies. Modeling DM halo as a degenerate fermion gas with the use of the stellar velocity dispersion data of $\mathrm{dSphs}$ and the inferred core size of the Fornax dwarf galaxy favor the fermion DM mass in the sub-keV range.

In this paper, we present a sub-keV fermion DDM scenario which addresses both the Hubble tension and the core-cusp problem. The model is based on a hidden Abelian gauge symmetry group and the fermion DDM is one of the chiral fermions required to cancel the chiral anomaly. The dark matter is nonthermally produced and sufficiently cooled down before virialization to follow the degenerate configuration. The Hubble tension is reconciled by the dark matter decaying into a massive dark photon and a light chiral fermion. We also comment about the constraint from the Lyman- $\alpha$ forest $[42,43]$ and show that our model is not inconsistent with its mass bound.

\section{A MODEL FOR THE DARK SECTOR}

Let us begin with the basic framework of our model for the dark sector. As a minimal extension of the gauge sector in a full theory, we consider a hidden Abelian gauge symmetry $U(1)_{X}$ under which matters in the dark sector are charged while SM particles are neutral.

As will be discussed below, the picture for the DDM solution to the Hubble tension in [13] assumes at least three particle candidates for (1) a decaying dark matter, (2) a radiation resulting from the decay, and (3) a daughter dark matter resulting from the decay. Thus, in the case where none of three is a SM particle, the introduction of fields in the dark sector other than the $U(1)_{X}$ gauge field is necessary. The anomaly-free condition for $U(1)_{X}$ is a useful guideline for how the introduction is made. As a matter of fact, a simple setup dubbed "Number Theory Dark Matter" for the dark sector along this line of logic was already discussed in $[44,45]$.

As for the total number of Weyl fermions $(N)$ and their $U(1)_{X}$ charges $\left(Q_{i}\right)$, anomaly-free conditions demand

$$
\sum_{i=1}^{N} Q_{i}^{3}=0, \quad \sum_{i=1}^{N} Q_{i}=0,
$$

for the cancellation of the $U(1)_{X}^{3}$ anomaly and gravitational $U(1)_{X} \times$ gravity $^{2}$ anomaly. If the theory allows the vectorlike fermions in the dark sector, the values of their mass parameters are naturally close to a cutoff scale. ${ }^{4}$ This point

\footnotetext{
${ }^{4}$ Of course, the mass of a vectorlike fermion is a free parameter and, in principle, it can be any value from zero to a UV cutoff of the theory. However, the small mass regime is less appealing from the theoretical point of view unless there is any reasonable supporting explanation like the restoration of certain symmetries in the zero limit.
}

makes the vectorlike fermions become irrelevant to the lowenergy physics once integrating out heavy degrees of freedom is done. Thus, we take an option to exclude the presence of the vectorlike fermions in the dark sector. As DM is massive, this option automatically requires the breaking of $U(1)_{X}$.

In order to have the particle contents required for the DDM scenario, we realize that the minimum number of Weyl fermions in the dark sector satisfying Eq. (1) amounts to five. ${ }^{5}$ Apart from the gauge field and the five Weyl fields, we introduce two complex scalar fields for the spontaneous breaking of $U(1)_{X}$ and to make fermions massive. Among several possibilities for a set of $U(1)_{X}$ charges discussed in $[44,45]$, we consider the following charge assignment:

$$
\psi_{-9}, \quad \psi_{-5}, \quad \psi_{-1}, \quad \psi_{7} \quad \psi_{8}, \quad \Phi_{1}, \quad \Phi_{6},
$$

where the subscripts denote $U(1)_{X}$ charges of each field. The first five are Weyl fields and the remaining two are complex scalars. One can see that the anomaly-free conditions in Eq. (1) are satisfied indeed by charges of Weyl fields. Writing the complex scalars as $\Phi_{1}=\phi_{1} e^{i A_{1} / V_{1}} / \sqrt{2}$ and $\Phi_{6}=\phi_{6} e^{i A_{6} / V_{6}} / \sqrt{2}$, we assume $V_{1} \equiv\left\langle\phi_{1}\right\rangle$ is greater than $V_{6} \equiv\left\langle\phi_{6}\right\rangle$ so that the breaking of $U(1)_{X}$ is induced dominantly by the condensation of $\Phi_{1}$ and hence the gauge boson mass is given by $m_{A}^{\prime} \simeq g V_{1}$, where $g$ is the gauge coupling constant. ${ }^{6}$

The Yukawa coupling of the dark sector reads

$$
\begin{aligned}
\mathcal{L}_{\text {Yuk }}= & y_{1} \Phi_{1} \psi_{-9} \psi_{8}+y_{2} \Phi_{6} \psi_{-5} \psi_{-1} \\
& +y_{3} \Phi_{6}^{\dagger} \psi_{-1} \psi_{7}+\text { H.c. },
\end{aligned}
$$

where $y_{i}(i=1-3)$ are taken to be real parameters without loss of generality. We diagonalize the mass matrix for $\psi_{-5}$, $\psi_{-1}$ and $\psi_{7}$ to obtain mass eigenstates. Together with $\psi_{-1}$, the following linear combination $\chi$ of $\psi_{-5}$ and $\psi_{7}$

$$
\chi \equiv\left(\frac{y_{2}}{\sqrt{y_{2}^{2}+y_{3}^{2}}}\right) \psi_{-5}+\left(\frac{y_{3}}{\sqrt{y_{2}^{2}+y_{3}^{2}}}\right) \psi_{7},
$$

forms a Dirac fermion $\Psi_{\mathrm{DM}}=\left(\psi_{-1}, \chi^{*}\right)^{T}$ of the mass $m_{\mathrm{DM}} \equiv \sqrt{y_{2}^{2}+y_{3}^{2}} V_{6}$. Also, the other orthogonal combination to $\chi$ forms a massless Weyl field $\xi$

\footnotetext{
${ }^{5}$ Three Weyl fermions cannot satisfy Eq. (1) due to the Fermat's theorem. Both cases of two and four Weyl fermions satisfying Eq. (1) give rise to vectorlike fermions, which is excluded in our option.

${ }^{6}$ To have values of $V_{1}$ and $V_{6}$ discussed in Sec. III, we suppress operators composed of both $\Phi_{1}$ and $\Phi_{6}$. To this end, as an exemplary way to do suppression as we desire, in an extra dimension picture we assume that the two complex scalars are localized in the different branes separated by a distance along an extra dimension which is large enough for suppressing effective interactions induced at $3+1$ dimensional spacetime.
} 


$$
\xi \equiv\left(\frac{y_{3}}{\sqrt{y_{2}^{2}+y_{3}^{2}}}\right) \psi_{-5}-\left(\frac{y_{2}}{\sqrt{y_{2}^{2}+y_{3}^{2}}}\right) \psi_{7} .
$$

From here on, we assume $y_{2}$ and $y_{3}$ are of a similar order and $y_{2} \simeq y_{3} \equiv y_{*}$ for simplicity.

\section{A CONCRETE SCENARIO}

In this section, we discuss how each resolution to phenomenological problems can fit into our model for the dark sector presented above. To have a simple thermal history of the dark sector, we first assume that the mass $\left(\simeq y_{1} V_{1}\right)$ of $\psi_{-9}$ and $\psi_{8}$ is greater than the inflaton mass $m_{I}$ so that we can neglect them in the cosmological history of the Universe. ${ }^{7}$

After the inflation ends, both the SM and the dark sector are reheated by the inflaton decay. As a $U(1)_{X}$ singlet, the inflaton $\Phi_{I}$ couples to both $\Phi_{1}$ and $\Phi_{6}$ through the following renormalizable operators ${ }^{8}$ :

$$
\mathcal{L}_{\Phi}=b_{1} \Phi_{I}\left|\Phi_{1}\right|^{2}+b_{6} \Phi_{I}\left|\Phi_{6}\right|^{2}
$$

where $b_{i}(i=1,6)$ is a dimensionful coupling coefficient. Assuming quartic couplings $\lambda_{1} \simeq \lambda_{6} \simeq \mathcal{O}(1)$ for each of complex scalars $\Phi_{1}$ and $\Phi_{6}$, we realize that decay of the inflaton to two $\phi_{1} \mathrm{~s}$ is kinematically suppressed because of $m_{I}<m_{1} \simeq \sqrt{\lambda_{1}} V_{1}$. Hence, for the dark sector, reheating is accomplished via inflaton decay to two $\phi_{6} \mathrm{~s}$. We define the decay rate to SM particles and the dark sector species $\left(\phi_{6}\right)$ to be $\Gamma_{\mathrm{SM}} \equiv \Gamma\left(\Phi_{I} \rightarrow \mathrm{SM}\right)$ and $\Gamma_{\mathrm{DS}} \equiv \Gamma\left(\Phi_{I} \rightarrow \phi_{6}+\phi_{6}\right)$, respectively, and make an approximation $\Gamma_{\text {tot }}=$ $\Gamma_{\mathrm{SM}}+\Gamma_{\mathrm{DS}} \simeq \Gamma_{\mathrm{SM}}$. Unless a reheating temperature is so large as to be close to the Planck scale, $\phi_{6}$ 's form a dark thermal bath via scattering with one another among them. Thus, below we consider the evolution of the dark thermal bath comprising purely $\phi_{6} \mathrm{~s} .{ }^{9}$ Notice that the purity of the thermal bath is guaranteed due to the smallness of both $y_{*}$ and $U(1)_{X}$ gauge coupling $(g)$, which will be shown later.

The concrete thermal history that we will show below as a viable physical scenario is the following. After the reheating era, the dark sector begins with the temperature

$$
T_{\mathrm{DS}}\left(a_{\mathrm{RH}}\right) \simeq 0.43 \times\left(\frac{m_{\mathrm{DM}}}{1 \mathrm{keV}}\right)^{-1 / 3} \times T_{\mathrm{RH}},
$$

where $T_{\mathrm{DS}}(a)\left(T_{\mathrm{SM}}(a)\right)$ denotes the temperature of the dark sector (the SM sector) at the time of the scale factor a. $a_{\mathrm{RH}}$ is the scale factor at the reheating time, and

\footnotetext{
${ }^{7} \mathrm{We}$ also assume that the reheating temperature $T_{\mathrm{RH}}$ is much less than the inflaton mass.

${ }^{8}$ In the extra dimension picture, taking the inflaton field $\Phi_{I}$ as the bulk field, we treat its coupling to $\Phi_{1}$ and $\Phi_{6}$ on an equal footing.

${ }^{9}$ In our work, we assume a negligibly small Higgs portal coupling between SM Higgs doublet and $\Phi_{6}$.
}

$T_{\mathrm{RH}} \equiv T_{\mathrm{SM}}\left(a_{\mathrm{RH}}\right)$ is assumed. ${ }^{10}$ From here on, whenever we encounter $T_{\mathrm{RH}}$, we can convert it using $\left(a_{\mathrm{RH}} T_{\mathrm{RH}}\right)=$ $\left(a_{\mathrm{EW}} T_{\mathrm{EW}}\right) \simeq 10^{-13} \mathrm{GeV}$ with $a_{\mathrm{EW}} \simeq 10^{-15}$ and $T_{\mathrm{SM}}\left(a_{\mathrm{EW}}\right) \simeq$ $100 \mathrm{GeV}$. Afterwards, the temperature of the dark thermal bath continues to decrease as

$$
T_{\mathrm{DS}}(a) \simeq 0.43 \times\left(\frac{m_{\mathrm{DM}}}{1 \mathrm{keV}}\right)^{-1 / 3} \times \frac{10^{-13}}{a} \mathrm{GeV}
$$

and becomes lower than the mass of $\phi_{6}$. The comoving number density of $\phi_{6}$ is preserved until the time when the rate of $\phi_{6}$ decay to a DM pair $\left(\psi_{-1}\right.$ and $\left.\chi\right)$ becomes comparable to the Hubble expansion rate, i.e., $\Gamma\left(\phi_{6} \rightarrow \mathrm{DM}+\mathrm{DM}\right) \simeq H$. Then, nonrelativistic $\phi_{6}$ 's start to decay to produce free-streaming DMs. The freestreaming of DM is ensured owing to the small $y_{*}$. From $\Gamma\left(\phi_{6} \rightarrow \mathrm{DM}+\mathrm{DM}\right) \simeq H$, we infer the temperature of the $\mathrm{SM}$ thermal bath at this time

$$
\begin{aligned}
T_{\mathrm{SM}}\left(a_{\mathrm{FS}}\right) \simeq & 537 \times g_{\mathrm{SM}}\left(a_{\mathrm{FS}}\right)^{-1 / 4} \\
& \times\left(\frac{V_{6}}{1 \mathrm{GeV}}\right)^{-1}\left(\frac{m_{\mathrm{DM}}}{1 \mathrm{keV}}\right) \sqrt{\frac{m_{6}}{1 \mathrm{GeV}}} \mathrm{GeV},
\end{aligned}
$$

where $a_{\mathrm{FS}}$ is the scale factor for the onset of the freestreaming of DM, $g_{\mathrm{SM}}(a)$ is the number of relativistic degrees of freedom in the SM sector evaluated at the scale factor $a$, and $m_{6}$ is the mass of $\phi_{6}$. Here we used $m_{\mathrm{DM}} \simeq y_{*} V_{6}$. Note that the decay of $\phi_{6}$ and the onset of DM's free-streaming take place at the same time since $\phi_{6}$ is nonrelativistic already at this time. For consistency, $T_{\mathrm{DS}}\left(a_{\mathrm{FS}}\right)$ should be less than $m_{6}$, which constrains $V_{6}\left(\simeq m_{6} / \sqrt{\lambda_{6}} \simeq m_{6}\right)$ for each $m_{\mathrm{DM}}$. In Fig. 2, the region above the green dashed line satisfies $T_{\mathrm{DS}}\left(a_{\mathrm{FS}}\right)<m_{6}$. Because of the entropy conservation, on the other hand, we can write down the temperature of the SM thermal bath as

${ }^{10}$ To get Eq. (7), the following relation is used:

$$
Y_{\mathrm{DM}} \equiv \frac{n_{\mathrm{DM}}}{s_{\mathrm{SM}}} \simeq 4.07 \times 10^{-4} \times\left(\frac{m_{\mathrm{DM}}}{1 \mathrm{keV}}\right)^{-1},
$$

where $n_{\mathrm{DM}}$ is the number density of the dark matter, $s_{\mathrm{SM}}$ is the entropy density of the SM sector, and we used the values, $\Omega_{\mathrm{DM}, 0}=0.24, \quad H_{0}=70 \mathrm{~km} / \mathrm{sec} / \mathrm{Mpc} \quad[13]$, and $s_{\mathrm{SM}, 0} \simeq$ $2.945 \times 10^{-11} \mathrm{eV}^{3}$ (entropy density today). Using the approximation $2 n_{\phi_{6}}=n_{\mathrm{DM}}$ at production time, one obtains

$$
\mathrm{Br} \frac{T_{\mathrm{RH}}}{m_{\Phi_{I}}} \simeq 2.7 \times 10^{-4} \times\left(\frac{m_{\mathrm{DM}}}{1 \mathrm{keV}}\right)^{-1},
$$

where $\mathrm{Br}$ is defined via $n_{6}=\mathrm{Br} \times n_{I} \simeq \mathrm{Br} \times\left(\rho_{\mathrm{SM}} / m_{I}\right)$ with $n_{6}$ and $n_{I}$ being the number density of $\phi_{6}$ and inflaton $\left(\Phi_{I}\right)$, respectively. 


$$
T_{\mathrm{SM}}(a)=\left(\frac{106.75}{g_{\mathrm{SM}}(a)}\right)^{1 / 3} \frac{10^{-13}}{a} \mathrm{GeV} .
$$

Equating Eq. (11) with Eq. (12) gives

$$
\begin{aligned}
a_{\mathrm{FS}}= & 8.8 \times 10^{-16} \times g_{\mathrm{SM}}\left(a_{\mathrm{FS}}\right)^{-1 / 12} \\
& \times\left(\frac{V_{6}}{1 \mathrm{GeV}}\right)\left(\frac{m_{\mathrm{DM}}}{1 \mathrm{keV}}\right)^{-1}\left(\frac{m_{6}}{1 \mathrm{GeV}}\right)^{-1 / 2},
\end{aligned}
$$

which will be used later for computing the free-streaming length for DM. After recombination, DM $\left(\Psi_{\mathrm{DM}}\right)$ gradually decays to a massless radiation $\xi$ and a $U(1)_{X}$ gauge boson $A_{\mu}^{\prime}$ serving as a daughter DM. On the other hand, due to a large redshift, DM gets into the motionless stage near the recombination era, completely behaving as a matter. Furthermore, its "would-be" temperature today without taking into account virialization due to gravity in the galaxy formation is sufficiently low to enable DM to be in a degenerate configuration.

In the following two subsections, we probe the parameter space of the model by applying the constraints on the lifetime of DDM and mass obtained in [13,36,37,39]. Eventually we show that there exists a parameter space where the desired physics can be realized in the model.

\section{A. Constraints from the Hubble Tension}

DDM solution to the Hubble tension is described by four parameters: lifetime of DDM, a fraction of a rest mass of DDM transferred to energy of the resulting radiation $(\epsilon)$, DM abundance today $\Omega_{\mathrm{DM}, 0} \equiv \rho_{\mathrm{DM}, 0} / \rho_{\mathrm{cr}, 0}$ and a reduced Hubble parameter $h \equiv H_{0} / \mathrm{Km} / \mathrm{sec} / \mathrm{Mpc}$ [13]. Below we refer to constraints on these parameters reported in [13] which were found by carrying out a Monte Carlo Markov Chain against the late universe measurements of the Hubble expansion rate for $0 \lesssim z \lesssim 2.4$. The underlying idea of the solution is to introduce a DDM whose decay leads to the production of a massless and a massive particle with four momenta $p_{\mu}=\left(\epsilon m_{\mathrm{DM}}, \vec{p}\right)$ and $p_{\mu}^{\prime}=\left((1-\epsilon) m_{\mathrm{DM}},-\vec{p}\right)$, respectively. A decrease in $\rho_{\mathrm{DM}} a^{3}$ after recombination due to the decay of DDM is compensated by an increase in $\rho_{\Lambda}$, which induces the earlier transition from the matterdominated era to the dark energy dominated era. In the end, $H(a)$ evolves to a larger $H_{0}$ value than that resulting from $\mathrm{CMB}$ based on $\Lambda \mathrm{CDM}$. As phenomenological consequences, enhancement of the late integrated Sachs-Wolfe effect for low $\ell$ regime $(\ell \sim 10)$ can be induced regarding the CMB temperature anisotropy power spectrum [46,47]. Also, the reduction of $f \sigma_{8}$ in comparison with that from $\Lambda \mathrm{CDM}$ and suppression of the kinetic Sunyaev-Zel'dovich power spectrum was further discussed in [47] as impacts that DDM can make. (Here $f$ is the matter growth rate, defined to be $f \equiv d \ln \delta_{m} / d \ln a$.)

In our model, we identify the Dirac fermion $\Psi_{\mathrm{DM}}=$ $\left(\psi_{-1}, \chi^{*}\right)^{T}$ generated from the decay of $\phi_{6}$ with DDM, the gauge boson of $U(1)_{X}$ with the resulting masssive particle, and the massless Weyl fermion $\xi$ with the resulting massless radiation. The dispersion relation of the resulting massive particle gives

$$
\frac{m_{A^{\prime}}}{m_{\mathrm{DM}}}=\sqrt{1-2 \epsilon} .
$$

From the coupling of the Dirac fermion DM to the gauge boson, the decay rate of DDM can be read as

$$
\begin{aligned}
\Gamma_{\mathrm{DM} \rightarrow A^{\prime} \xi} & =\frac{9}{4 \pi} \epsilon g^{2} m_{A^{\prime}}\left[\left(\frac{m_{\mathrm{DM}}}{m_{A^{\prime}}}\right)^{3}-2 \frac{m_{A^{\prime}}}{m_{\mathrm{DM}}}+\frac{m_{\mathrm{DM}}}{m_{A^{\prime}}}\right] \\
& =\frac{9 g^{2} m_{A^{\prime}}}{2 \pi} \times \frac{\epsilon^{2}(3-4 \epsilon)}{(1-2 \epsilon)^{3 / 2}} .
\end{aligned}
$$

Applying $1 \sigma$ level constraints $1.376 \times 10^{-43} \mathrm{GeV} \lesssim$ $\Gamma_{\mathrm{DM} \rightarrow A^{\prime} \xi} \lesssim 1.04 \times 10^{-42} \mathrm{GeV}^{11}$ and $0.0013 \lesssim \epsilon \lesssim 0.229$ [13] to Eq. (15), we obtain the constraints on $\left(g, V_{1}\right)$ which is shown as the yellow shaded region in Fig. 1. The right panel is the magnification of the left panel for the smaller coupling regime where not only the $1 \sigma$ but also the $2 \sigma$ level is shown as a green shaded region (the region above the green solid line). ${ }^{12}$

\section{B. Constraints from the core-cusp problem}

Because of DM's fermionic nature, provided that a thermal history of DM can allow for its degenerate configuration near the time of the structure formation, then our DM candidate may form cored density profiles in the Milky Way's dSphs satellites by balancing the attractive gravitational force with the repulsive Fermi quantum pressure. To see whether our DM can go through a sufficient redshift for achieving the degenerate configuration, we need to estimate DM's wouldbe temperature today, $\tilde{T}_{\mathrm{DM}, 0}$, in the absence of a structure formation and compare it to a degeneracy temperature, $T_{\mathrm{DEG}}$, for dSphs to check if $\tilde{T}_{\mathrm{DM}, 0}$ is less than $T_{\mathrm{DEG}}$ of dSphs [37]. Starting with $T_{\mathrm{DM}}\left(a_{\mathrm{FS}}\right) \simeq m_{6} / 2$, we obtain $T_{\mathrm{DM}}\left(a_{\mathrm{NR}}\right) \simeq$ $\left(m_{6} a_{\mathrm{FS}}\right) /\left(2 a_{\mathrm{NR}}\right) \simeq m_{\mathrm{DM}}$ where $a_{\mathrm{NR}}$ is the scale factor when DM becomes nonrelativistic. Combined with Eq. (13), this yields

$$
\begin{aligned}
a_{\mathrm{NR}}= & 4.4 \times 10^{-10} \times g_{\mathrm{SM}}\left(a_{\mathrm{FS}}\right)^{-1 / 12} \\
& \times\left(\frac{V_{6}}{1 \mathrm{GeV}}\right)\left(\frac{m_{\mathrm{DM}}}{1 \mathrm{KeV}}\right)^{-2}\left(\frac{m_{6}}{1 \mathrm{GeV}}\right)^{1 / 2} .
\end{aligned}
$$

Since $a=a_{\mathrm{NR}}, T_{\mathrm{DM}}$ scales as $\sim a^{-2}$, implying that

\footnotetext{
${ }^{11}$ The constraint on the DDM lifetime discussed in Refs. $[48,49]$ is not applied here because DDM only decaying into dark radiations is assumed there.

${ }^{12}$ The lifetime of $A^{\prime}$ can be longer than that of $\Psi_{\mathrm{DM}}$ depending on $y_{2}$ and $y_{3}$.
} 

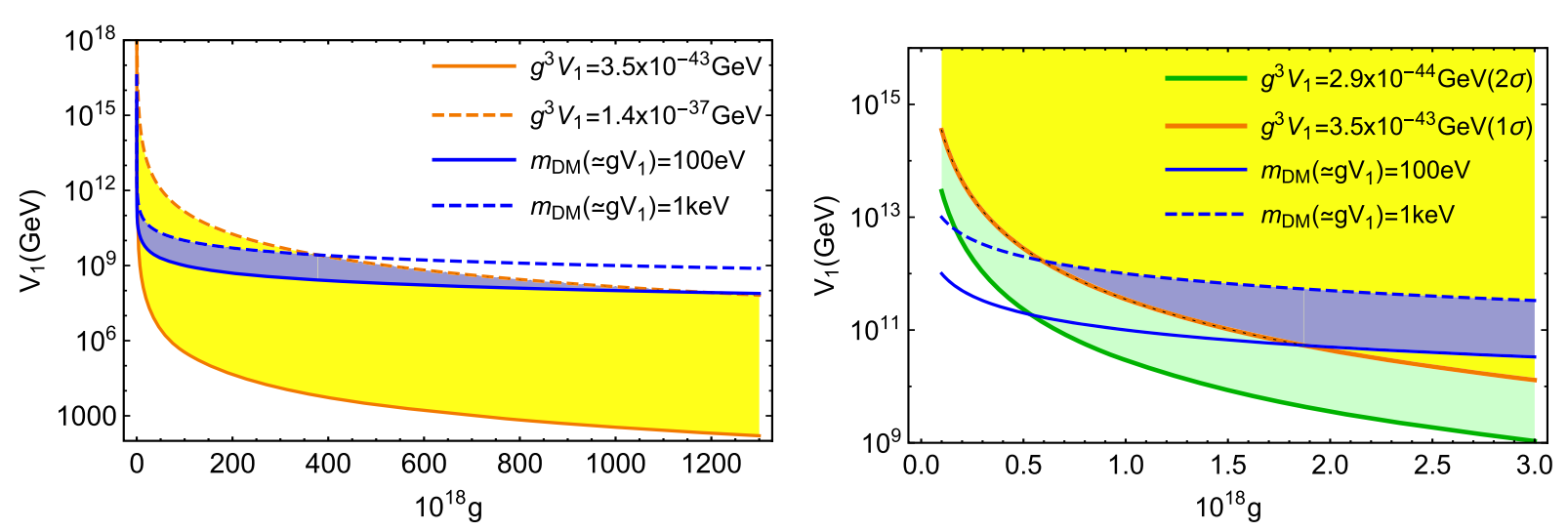

FIG. 1. Constraints on $\left(g, V_{1}\right)$ space where $g$ is the gauge coupling of $U(1)_{X}$. The right panel is a magnification of the left panel for the relatively smaller coupling regime. The yellow shaded region between the yellow solid and dashed lines is mapping onto the $\left(g, V_{1}\right)$ space of constraints on the life of DDM and the $\epsilon$ parameter in the DDM solution to the Hubble tension [13] at $1 \sigma$ level. (On the right panel, we showed the same mapping at $2 \sigma$ level with the green solid line and green shaded region.) The blue shaded region corresponds to $\left(g, V_{1}\right)$ values that produce a sub-keV decaying fermion DM as a solution to not only the Hubble tension, but also the core-cusp problem, satisfying $1 \sigma$ level constraints.

$$
\tilde{T}_{\mathrm{DM}, 0} \simeq \frac{m_{6} a_{\mathrm{FS}}}{2 a_{\mathrm{NR}}}\left(\frac{a_{\mathrm{NR}}}{a_{0}}\right)^{2}
$$

where $a_{0}=1$ is the scale factor today. Below we will see values of $V_{1}$ obtained from the free-streaming length constraint lie in $V_{6} \simeq \mathcal{O}(10)-\mathcal{O}(100) \mathrm{GeV}$. Using Eq. (16) and Eq. (17) with $V_{6} \simeq \mathcal{O}(10)-\mathcal{O}(100) \mathrm{GeV}$, we estimate would-be temperature today of sub-keV DM to obtain $\mathcal{O}\left(10^{-14}\right)-\mathcal{O}\left(10^{-11}\right) \mathrm{eV}$. This corresponds to $\mathcal{O}\left(10^{-10}\right) \mathrm{K}-\mathcal{O}\left(10^{-6}\right) \mathrm{K}$, which is lower than the degeneracy temperature for dSphs $T_{\mathrm{DEG}} \simeq \mathcal{O}\left(10^{-4}\right) \mathrm{K}-\mathcal{O}\left(10^{-3}\right) \mathrm{K}$ [37]. Hence, it is confirmed that our DM is capable of achieving the degenerate configuration today when the heat-up due to the virialization is neglected.

As such, the mass of our DM is subject to the TremaineGunn bound [50] which arises due to the presence of the maximum phase space density of the degenerate Fermi gas in a galaxy. When applied to dSphs, for a generic fermion DM, the logic underlying the Tremaine-Gunn bound gives $m_{\mathrm{DM}, \min } \sim 70-300 \mathrm{eV}$ as a lower bound on mass [39,51]. Also, recently, a fitting analysis for the stellar kinematics of the relatively smaller dSphs (Leo II, Willman I, Segue I) set a conservative mass lower bound $\sim 100 \mathrm{eV}$ [52]. ${ }^{13}$ In light of these results, we focus on the fermion DM mass greater than $\sim 100 \mathrm{eV}$ in our model. Combined with a constraint on the $\epsilon$ parameter in the DDM solution to the Hubble tension, Eq. (14) tells us that $m_{A^{\prime}}$ is at least $70 \%$ of $m_{\mathrm{DM}}$. Thus, we may approximate $m_{\mathrm{DM}} \simeq m_{A^{\prime}}=g V_{1}$

\footnotetext{
${ }^{13}$ These small size galaxies are particularly important for studying the possibility of a degenerate fermion DM and its mass since an increase in the DM temperature due to virialization is minimized. For large size galaxies, the fermion DM would be present there in nondegenerate configurations, which makes inferring the fermion DM mass from the stellar kinematics of the large size galaxies more difficult.
}

and apply $m_{\mathrm{DM}}>100 \mathrm{eV}$ to the $\left(g, V_{1}\right)$ plane to improve the constraints obtained in Sec. III A. In Fig. $1, m_{\mathrm{DM}}=100 \mathrm{eV}$ is represented as the blue solid line. Thereby for the right panel, the allowed parameter space at $1 \sigma(2 \sigma)$ level reduces to the subarea of the yellow (green) colored region which intersects with the region above the blue solid line.

Next, in order for the degenerate fermion DM to serve as a solution to the core-cusp problem, it cannot have too large a mass because the core size of the fermion DM density profile tends to decrease when $m_{\mathrm{DM}}$ increases. In this context, there have been efforts to constrain fermion DM mass by using the kinematic of dSphs. In [37], the use of a fermion DM density profile obtained by solving the LaneEmden equation achieves a good fit to the stellar velocity dispersion of eight classical dwarf galaxies especially for the DM mass regime 100-200 eV. ${ }^{14}$ In addition, in [39], the use of the core size of the Fornax dSphs gives $70 \mathrm{eV} \lesssim$ $m_{\mathrm{DM}} \lesssim 400 \mathrm{eV}$ under the assumption of quasidegenerate Fermi gas as DM. In contrast, the relatively large fermion DM mass near 1-2 keV was pointed out in [36] by requiring the mass of $\mathrm{dSph}$ Willman I be greater than the minimum halo mass made up of the degenerate fermion DM. Given these arguments for $m_{\mathrm{DM}}$, in this work we concentrate on sub-keV fermion DM mass regime, i.e., $m_{\mathrm{DM}}<1 \mathrm{keV}$. Similarly to the previous section, we apply $m_{\mathrm{DM}} \lesssim 1 \mathrm{keV}$ to the $\left(g, V_{1}\right)$ plane to get the blue dashed line and area below it in Fig. 1. As the final intersection of several constraints at $1 \sigma$ level discussed so far, the blue shaded region in Fig. 1 is obtained, which allows our model to produce a degenerate decaying

\footnotetext{
${ }^{14}$ The Lane-Emden equation results from combining the continuity equation and the hydrostatic equilibrium equation with the Fermi pressure.
} 
fermion DM resolving the Hubble tension and the corecusp problem. ${ }^{15}$

Intriguingly, we observe that the blue shaded region $(1 \sigma)$ in Fig. 1 can cover the $V_{1}$ value up to $10^{12} \mathrm{GeV}$ for the gauge coupling as small as $g \simeq 10^{-18}$. Besides, when the $2 \sigma$ level constraints on the $\left(g, V_{1}\right)$ plane are considered, even the $V_{1}$ value as large as $10^{13} \mathrm{GeV}$ is allowed. As we mentioned above, $V_{1}$ is assumed to be larger than the inflaton mass $m_{I}$ in the model. This implies that the model can be consistent with even the high scale inflation models such as chaotic inflation [53] or topological inflation [54] where the inflaton mass is $m_{I} \simeq 10^{13} \mathrm{GeV}$.

\section{Constraints from the free-streaming length}

Here we discuss constraints on the Yukawa coupling $y_{*}$ as well as $V_{6}\left(\simeq m_{6}\right)$. As a decay product of a nonrelativistic $\phi_{6}$, which was never in a thermal equilibrium with other species, our Dirac fermion DM $\left(\Psi_{\mathrm{DM}}\right)$ is expected to follow the delta-function-like distribution in its momentum space, centered on $|\vec{p}|=m_{6} / 2$ at $a=a_{\mathrm{FS}}$. From then on, DM becomes nonrelativistic around the time $a=a_{\mathrm{NR}}$ given in Eq. (16). Bearing in mind $a_{\mathrm{BBN}} \simeq 10^{-10}-10^{-9}$, we realize that for sub-keV DM, $V_{6}\left(\simeq m_{6}\right)$ around the GeV scale or greater than that makes DM still relativistic at the big bang nucleosynthesis $(\mathrm{BBN})$ era. DM may contribute to the energy budget of the Universe at the BBN era as a radiation. Therefore, we apply $\Delta N_{\text {eff }}^{\mathrm{BBN}} \lesssim 1$ [55] to the DM energy density at the BBN era to constrain $V_{6}$. Using Eq. (8) and Eq. (13) with

$\rho_{\mathrm{DM}}\left(a_{\mathrm{BBN}}\right)=\sqrt{m_{\mathrm{DM}}^{2}+\left(\frac{m_{6} a_{\mathrm{FS}}}{2 a_{\mathrm{BBN}}}\right)^{2}} Y_{\mathrm{DM}} s_{\mathrm{SM}}\left(a_{\mathrm{BBN}}\right)$,

yields the parameter space in the $\left(m_{\mathrm{DM}}, V_{6}\right)$ plane satisfying the condition $\Delta N_{\text {eff }}^{\mathrm{BBN}} \lesssim 1$. In Fig. 2, this corresponds to the region below the black dashed line.

Given the constraint on a value of $V_{6}$ around $\mathcal{O}(10)_{-}$ $\mathcal{O}(100) \mathrm{GeV}$ (the region between the black and green dashed lines in Fig. 2), it becomes clear that sub-keV DM is produced from the $\phi_{6}$ decay as a relativistic particle. So the next question that should be further asked is whether DM in the model can be consistent with Lyman- $\alpha$ forest data or not. Should the free-streaming length $\left(\lambda_{\mathrm{FS}}\right)$ of DM be too large, the structure formation at the scale below $\lambda_{\mathrm{FS}}$ would have been erased, likely to cause a disagreement with the nonvanishing matter power spectrum at the large scales. On the contrary, if $\lambda_{\mathrm{FS}}$ is too short (i.e., $\lambda_{\mathrm{FS}}<0.1 \mathrm{Mpc}$ ), the DM model tends to produce too many satellite galaxies of Milky and Andromeda as opposed to the observed number. Therefore, we require $0.3 \mathrm{Mpc}<\lambda_{\mathrm{FS}}<0.5 \mathrm{Mpc}$ to further constrain $V_{6}[37,56-58]$.

\footnotetext{
${ }^{15}$ In the right panel, the intersection coming from constraints at the $2 \sigma$ level is the subspace between the blue solid and dashed lines which overlaps the green shaded region above the green solid line.
}

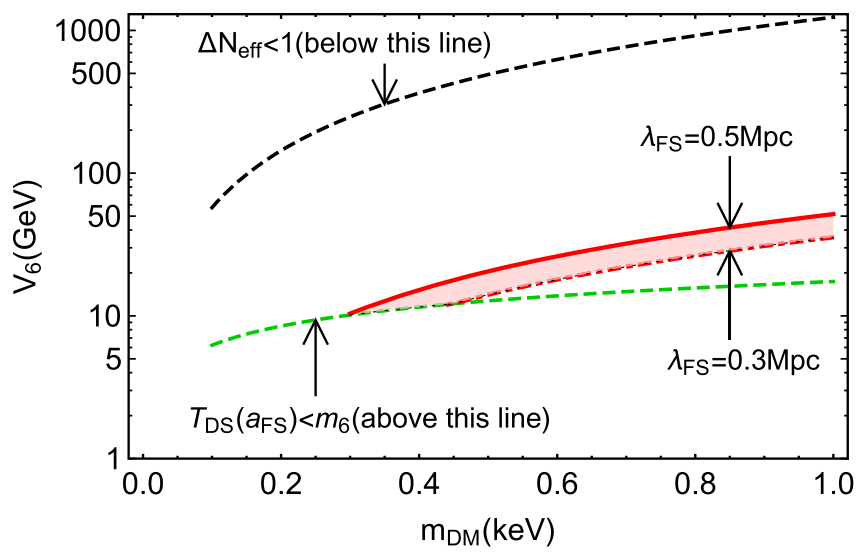

FIG. 2. $\left(m_{\mathrm{DM}}, V_{6}\right)$ plane which is constrained by (1) $T_{\mathrm{DS}}\left(a_{\mathrm{FS}}\right)<m_{6}$ (region above the green dashed line), (2) $\Delta N_{\text {eff }}^{\mathrm{BBN}} \lesssim 1$ (region below the black dashed line), and (3) $0.3 \mathrm{Mpc}<\lambda_{\mathrm{FS}}<0.5 \mathrm{Mpc}$ (region between the solid and dot-dashed red lines). The eventual intersection of these lies is in the red colored shaded region.

The free-streaming length is given by

$$
\begin{aligned}
\lambda_{\mathrm{FS}} & =\int_{t_{\mathrm{FS}}}^{t_{0}} \frac{v(t)}{a(t)} d t \\
& \simeq \int_{a_{\mathrm{FS}}}^{1} \frac{\left(a_{\mathrm{FS}} m_{6} / H_{0}\right) d a}{\sqrt{\Omega_{\mathrm{rad}, 0}+a \Omega_{\mathrm{m}, 0}} \sqrt{\left(2 a m_{\mathrm{DM}}\right)^{2}+\left(a_{\mathrm{FS}} m_{6}\right)^{2}}},
\end{aligned}
$$

where $v(t)$ is the DM velocity, and $\Omega_{\mathrm{rad}, 0}, \Omega_{\mathrm{m}, 0}$ denote the radiation and matter density parameter, respectively. The dependence of $V_{6}$ appears through $a_{\mathrm{FS}}$ in Eq. (13). We show the result of mapping $0.3 \mathrm{Mpc}<\lambda_{\mathrm{FS}}<0.5 \mathrm{Mpc}$ to the $\left(m_{\mathrm{DM}}, V_{6}\right)$ plane in Fig. 2 as the space between the two red solid and dot-dashed lines. The two red lines correspond to each of $\lambda_{\mathrm{FS}}=0.5$ and $0.3 \mathrm{Mpc}$. The eventual intersection in the $\left(m_{\mathrm{DM}}, V_{6}\right)$ plane reflecting four constraints discussed so far is shown as the red shaded region in Fig. 2. For DM with mass $100-300 \mathrm{eV}$, we found that there is no parameter space that allows DM to travel the smaller free-streaming length than $0.5 \mathrm{Mpc}$. In contrast, we found that there exists indeed a consistent parameter space for the mass regime $\sim 300-1000 \mathrm{eV}$. The strength of the Yukawa $y_{*}$ associated with this $V_{6}$ regime reads $10^{-8}-10^{-7}$. This may appear to be in tension with [37,39]. However, note that to resolve the core-cusp problem with $100 \mathrm{eV} \lesssim$ $m_{\mathrm{DM}} \lesssim 400 \mathrm{eV}$, either of a fully or quasi degenerate fermion DM configuration was assumed in $[37,39]$ in the absence of considering a baryonic effect in classical dSphs. As was pointed out in [39], when additional effects such as baryonic feedback or nontrivial DM momentum distribution skewed to lower energies are taken into account together with Fermi repulsion, the upper bound of $m_{\mathrm{DM}}$ might be alleviated. Additionally, in our work, we expect the actual momentum distribution of our DM on production to have a broader width due to nonzero velocity dispersion of DM when produced and a center smaller than $m_{6} / 2$, although we approximated 
it as a delta-function-like peak with the assumption of instantaneous decay of $\phi_{6}$. This consideration may help some parts of $100 \mathrm{eV} \lesssim m_{\mathrm{DM}} \lesssim 300 \mathrm{eV}$ in Fig. 2 produce the smaller $\lambda_{\mathrm{FS}}$ than we obtained in the present analysis.

\section{DISCUSSION}

In this article, we present a particle physics model which describes a completely isolated dark sector lacking any way to communicate with the SM sector. The dark sector enjoys an Abelian gauge symmetry $U(1)_{X}$ which meets the anomalyfree conditions solely due to fields in the dark sector. We assume $U(1)_{X}$ without the kinetic mixing with $U(1)_{\mathrm{EM}}$ in the $\mathrm{SM}$. This setup trivially does not affect any known experimental result in the SM. With the purpose to keep the model minimal and consistent with the decaying DM solution to the Hubble tension, we introduced two darkly charged complex scalars and five darkly charged Weyl fermions along with the $U(1)_{X}$ gauge boson. Starting from an inflation model with inflaton mass smaller than the $U(1)_{X}$ breaking scale, we showed that one linear combination of two of five Weyl fermions with sub-keV mass can play a role of DM which starts to decay after recombination with the lifetime $\sim 35 \mathrm{Gyr}$. Within the parameter space of the model we found, the redshift that the DM candidate experiences since its production from the decay of a complex scalar makes the would-be temperature of DM today without virialization low enough to form a cored density profile for the Milky Way's dwarf spheroidal satellites. The main conclusion in this paper is based on the DM production mechanism. Although we restrict our discussion to the specific model, the similar conclusions regarding the free-streaming length of DM will be obtained as long as a DM production mechanism presented in this work is used.

As such, the DM candidate in our model is expected to serve as a solution to both the Hubble tension and the core-cusp problem. Originating from the dark sector lacking a coupling to the SM sector, our DM candidate interacts with the SM particles only via gravitational interaction. We found that for resolving the Hubble tension, DM needs to decay via the gauge interaction with the coupling as tiny as $\sim 10^{-18}$ $10^{-15}$ for having the required long life time. Interestingly, it turns out in the model that the strength of $U(1)_{X}$ gauge coupling is also linked to the a scale of consistent inflation models. Particularly, inflation models with an inflaton mass as large as $10^{13} \mathrm{GeV}$ require the gauge coupling as small as $10^{-18}$. For the sub-keV DM mass regime required to solve the core-cusp problem, this coupling strength is still larger than the strength of the gravitational interaction that DM does, i.e., $\sim m_{\mathrm{DM}} / M_{P} \simeq 10^{-24}$. As for the sub-keV DM mass regime, we found that the DM mass allowed in the model is in a slight tension with what is required for a degenerate fermion DM solution to the core-cusp problem, which we believe can be alleviated by a more detailed analysis about the phase-space distribution of DM and the use of unknown baryonic effects on dSphs galaxy formation. ${ }^{16}$

\section{ACKNOWLEDGMENTS}

T. T. Y. is supported in part by the China Grant for Talent Scientific Start-Up Project and the JSPS Grant-inAid for Scientific Research No. 16H02176, No. 17H02878, and No. $19 \mathrm{H} 05810$ and by World Premier International Research Center Initiative (WPI Initiative), MEXT, Japan.

\footnotetext{
${ }^{16}$ Another possibility to alleviate the tension is to produce the fermion DM nonthermally by the inflaton decay. In this case, even the dark matter mass as low as $m_{\mathrm{DM}} \simeq 200 \mathrm{eV}$ can achieve a short enough free-streaming length [37]. We apply a similar nonthermal DM production mechanism to a future study within the present framework [59].
}

[1] W. Hu, R. Barkana, and A. Gruzinov, Phys. Rev. Lett. 85, 1158 (2000).

[2] L. Hui, J. P. Ostriker, S. Tremaine, and E. Witten, Phys. Rev. D 95, 043541 (2017).

[3] K. Inomata, M. Kawasaki, K. Mukaida, Y. Tada, and T. T. Yanagida, Phys. Rev. D 96, 043504 (2017).

[4] L. Verde, T. Treu, and A. G. Riess, Nat. Astron. 3, 891 (2019).

[5] A. G. Riess et al., Astrophys. J. 826, 56 (2016).

[6] A. G. Riess et al., Astrophys. J. 861, 126 (2018).

[7] V. Bonvin et al., Mon. Not. R. Astron. Soc. 465, 4914 (2017).

[8] S. Birrer et al., Mon. Not. R. Astron. Soc. 484, 4726 (2019).

[9] N. Aghanim et al. (Planck Collaboration), arXiv:1807.06209.
[10] G. Efstathiou, Mon. Not. R. Astron. Soc. 440, 1138 (2014).

[11] W. L. Freedman, Nat. Astron. 1, 121 (2017).

[12] M. Rameez and S. Sarkar, arXiv:1911.06456.

[13] K. Vattis, S. M. Koushiappas, and A. Loeb, Phys. Rev. D 99, 121302 (2019).

[14] G. Choi, M. Suzuki, and T. T. Yanagida, arXiv:1910.00459.

[15] J. L. Feng, A. Rajaraman, and F. Takayama, Phys. Rev. Lett. 91, 011302 (2003).

[16] J. L. Bernal, L. Verde, and A. G. Riess, J. Cosmol. Astropart. Phys. 10 (2016) 019.

[17] S. Vagnozzi, arXiv:1907.07569.

[18] T. Karwal and M. Kamionkowski, Phys. Rev. D 94, 103523 (2016).

[19] V. Poulin, T. L. Smith, T. Karwal, and M. Kamionkowski, Phys. Rev. Lett. 122, 221301 (2019). 
[20] S. Alexander and E. McDonough, Phys. Lett. B 797, 134830 (2019).

[21] J. Sakstein and M. Trodden, arXiv:1911.11760.

[22] W. Yang, S. Pan, E. Di Valentino, R. C. Nunes, S. Vagnozzi, and D. F. Mota, J. Cosmol. Astropart. Phys. 09 (2018) 019.

[23] E. Di Valentino, A. Melchiorri, O. Mena, and S. Vagnozzi, arXiv:1908.04281.

[24] B. Moore, T. R. Quinn, F. Governato, J. Stadel, and G. Lake, Mon. Not. R. Astron. Soc. 310, 1147 (1999).

[25] B. Moore, S. Ghigna, F. Governato, G. Lake, T. R. Quinn, J. Stadel, and P. Tozzi, Astrophys. J. 524, L19 (1999).

[26] S. Y. Kim, A. H. G. Peter, and J. R. Hargis, Phys. Rev. Lett. 121, 211302 (2018).

[27] M. Boylan-Kolchin, J. S. Bullock, and M. Kaplinghat, Mon. Not. R. Astron. Soc. Lett. 415, L40 (2011).

[28] D. H. Weinberg, J. S. Bullock, F. Governato, R. K. de Naray, and A. H. G. Peter, Proc. Natl. Acad. Sci. U.S.A. 112, 12249 (2015).

[29] J. F. Navarro, C. S. Frenk, and S. D. M. White, Astrophys. J. 490, 493 (1997).

[30] T. Fukushige and J. Makino, Astrophys. J. 477, L9 (1997).

[31] T. Ishiyama, J. Makino, S. P. Zwart, D. Groen, K. Nitadori, S. Rieder, C. de Laat, S. McMillan, K. Hiraki, and S. Harfst, Astrophys. J. 767, 146 (2013).

[32] A. Borriello and P. Salucci, Mon. Not. R. Astron. Soc. 323, 285 (2001).

[33] G. Gilmore, M. I. Wilkinson, R. F. G. Wyse, J. T. Kleyna, A. Koch, N. W. Evans, and E. K. Grebel, Astrophys. J. 663, 948 (2007).

[34] S.-H. Oh, W. J. G. de Blok, F. Walter, E. Brinks, and R. C. Kennicutt, Jr., Astron. J. 136, 2761 (2008).

[35] W. J. G. de Blok, Adv. Astron. 2010, 789293 (2010).

[36] C. Destri, H. J. de Vega, and N. G. Sanchez, New Astron. 22, 39 (2013).

[37] V. Domcke and A. Urbano, J. Cosmol. Astropart. Phys. 01 (2015) 002.

[38] S. Alexander and S. Cormack, J. Cosmol. Astropart. Phys. 04 (2017) 005.
[39] L. Randall, J. Scholtz, and J. Unwin, Mon. Not. R. Astron. Soc. 467, 1515 (2017).

[40] B. G. Giraud and R. Peschanski, Phys. Scr. 94, 085003 (2019).

[41] D. Savchenko and A. Rudakovskyi, Mon. Not. R. Astron. Soc. 487, 5711 (2019).

[42] M. Viel, G. D. Becker, J. S. Bolton, and M. G. Haehnelt, Phys. Rev. D 88, 043502 (2013).

[43] V. Iršič et al., Phys. Rev. D 96, 023522 (2017).

[44] K. Nakayama, F. Takahashi, and T. T. Yanagida, Phys. Lett. B 699, 360 (2011).

[45] K. Nakayama, F. Takahashi, and T. T. Yanagida, Phys. Lett. B 790, 218 (2019).

[46] V. Poulin, P. D. Serpico, and J. Lesgourgues, J. Cosmol. Astropart. Phys. 08 (2016) 036.

[47] L. Xiao, L. Zhang, R. An, C. Feng, and B. Wang, J. Cosmol. Astropart. Phys. 01 (2020) 045.

[48] K. Enqvist, S. Nadathur, T. Sekiguchi, and T. Takahashi, J. Cosmol. Astropart. Phys. 09 (2015) 067.

[49] K. Enqvist, S. Nadathur, T. Sekiguchi, and T. Takahashi, arXiv: 1906.09112.

[50] S. Tremaine and J. E. Gunn, Phys. Rev. Lett. 42, 407 (1979).

[51] A. Boyarsky, O. Ruchayskiy, and D. Iakubovskyi, J. Cosmol. Astropart. Phys. 03 (2009) 005.

[52] C. Di Paolo, F. Nesti, and F. L. Villante, Mon. Not. R. Astron. Soc. 475, 5385 (2018).

[53] K. Nakayama, F. Takahashi, and T. T. Yanagida, Phys. Lett. B 730, 24 (2014).

[54] K. Harigaya, M. Kawasaki, and T. T. Yanagida, Phys. Lett. B 719, 126 (2013).

[55] G. Mangano and P. D. Serpico, Phys. Lett. B 701, 296 (2011).

[56] F. Borzumati, T. Bringmann, and P. Ullio, Phys. Rev. D 77, 063514 (2008).

[57] J. A. R. Cembranos, J. L. Feng, A. Rajaraman, and F. Takayama, Phys. Rev. Lett. 95, 181301 (2005).

[58] P. Colin, V. Avila-Reese, and O. Valenzuela, Astrophys. J. 542, 622 (2000).

[59] G. Choi, M. Suzuki, and T. T. Yanagida (to be published). 